\title{
Moving North But Never Far From Us
}

\author{
Yongheng Wang ${ }^{1}$, Yuxiao $\mathrm{Li}^{2}$, Yuewen Yang ${ }^{2}$, Zhifan Zhang ${ }^{3}$ \\ ${ }^{1}$ School of Electric Power Engineering, South China University of Technology, Guangzhou, Guangdong, \\ 510006, China. \\ ${ }^{2}$ School of Economics and Commerce, South China University of Technology, Guangzhou, Guangdong, \\ 510006, China. \\ ${ }^{3}$ School of Electronic and Information Engineeing, South China University of Technology, Guangzhou, \\ Guangdong, 510006, China.
}

Keywords: Time-Series; Analytic Hierarchy Process; Support Vector Machine.

\begin{abstract}
Over the past 20 years, the way North Atlantic mackerel and herring migrate has changed dramatically. In the 1980s, the migration occurred in the late summer and autumn. Now, the start of the migration is lagging, and their migration routes move further offshore. Firstly, we establish a support vector machine (SVM) model to predict the temperature of a single geographic coordinate point, and then applied it to the whole North Atlantic Ocean. After predicting the sea surface temperatures in the next 50 years, we conduct cluster analysis using ARCGIS, the geographic information analysis tool, to optimize our SVM model. We find that 10 isotherm gradually move northward in the next 50 years, specifically, the habitat of the two fish species will move $2.5 \circ$ northward in the future, further away from the mainland. Secondly, we design an operation evaluation model for a small fisheries company based on previous forecast, and assume the best scenario, that is, the rate of global warming to maintain the status QUO, and the worst scenario, 1.5 times faster global warming speed. Our model shows that in the best case, it is unprofitable for the company continuously go fishing after 45 years, and in the worst case, only 5 years. Finally, given that fishing places might move to another country, and give constructive operation suggestions like fishing by time, by categories and by quota.
\end{abstract}

\section{Introduction}

The ocean is the main body of the earth, cradle of life, and source of human civilization. However, global warming has caused tremendous damage to the marine environment over the last decades. The increase in atmospheric temperature has changed atmospheric circulation, posting a threat to the ocean current and causing the dramatic rise of surface temperature of ocean, the rapid melting of glaciers, the climb of sea level, and the disappearance of some coastal lowlands. These influences will then transform the living habits of some marine life through change the locations of their food resources and living environment, and they have no choice but to migrate, in order to find a more suitable place to reproduce and survive.

\section{Migration Location Prediction Model Based on Support Vector Regression (Svr)}

\subsection{The Construction of the Model}

From the NASA database, we obtained official data on temperature changes in the North Atlantic Ocean over the past 20 years(in Appendix). Then through the fitting analysis of the temperature data of the North Atlantic Ocean in over the past 20 years, we can predicate the temperature change in this area in the next 50 years, and then find out the most likely migration locations of herring and mackerel in the next 50 years, so we introduce vector regression (SVR). Firstly,we define the loss function as follows:

$$
\operatorname{erroy}(y, s)=\max (0,|s-y|-\epsilon)
$$


where $\epsilon$ is the height of the temperature margin. The data falling within the height range within 20 years is set as the ideal temperature data, and the error outside is $|\gamma-\mathrm{S}|-\epsilon$, and $\gamma$ represents the value of the ideal temperature fitting curve. $\mathrm{S}$ represents the value $\mathrm{y}$ of the observed temperature sample. The figure for the loss function is as follow:

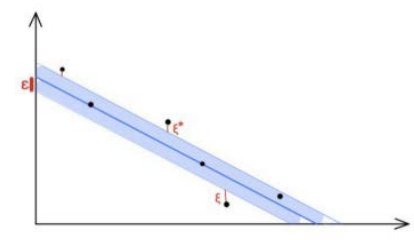

Figure1 The schematic diagram of the loss function

In order to reduce the over fitting of the model, we add a regularization term $\mathrm{L}$. The regularization term $\mathrm{L}$ reduces the over fitting of the model by increasing the constraints of the model parameters. The regular expression $\mathrm{L}$ in the form of Lagrange multiplier method as shown below:

$$
\begin{gathered}
L=\frac{1}{2} w^{T} w+C \sum_{n=1}^{N}\left(\xi^{*} n+\xi n\right)-\sum_{n=1}^{N} a^{8} i\left(\epsilon+\xi^{*} n-y^{i}+w^{T} z^{i}+b\right) \\
-\sum_{n=1}^{N} a^{i}\left(\epsilon+\xi^{i}+y^{i}-w^{T} z^{i}-b\right)-\sum_{n=1}^{N}\left(\eta^{i} \xi^{i}-b\right)-\sum_{n=1}^{N}\left(\eta^{i} \xi^{i}+\eta^{*} i+\eta^{*} \xi^{*}\right) \\
\frac{\partial L}{\partial b}=\sum_{i=1}^{N}\left(a_{i}^{*}-a_{i}\right)=0 \\
\frac{\partial L}{\partial w}=w-\sum_{i=1}^{N}\left(a_{i}^{*}-a_{i}\right) z^{i}=0 \\
\frac{\partial L}{\partial \xi_{i}^{8}}=C-a_{i}^{*}-\eta_{i}^{*}=0
\end{gathered}
$$

The solution of the above is a standard quadratic planning problem. We can simplify it to get the following formulas:

$$
\begin{aligned}
& a_{i}^{*}\left(\epsilon+\xi_{i}^{*}-y_{i}+w^{T} z_{i}+b\right)=0 \\
& a_{i}^{*}\left(\epsilon+\xi_{i}+y_{i}+w^{T} z_{i}-b\right)=0 \\
& w=\sum_{i=1}^{N}\left(a_{i}-a_{i}^{*}\right) z i
\end{aligned}
$$

Where we can see that if the point falls within the area of margin, then both $\xi i *$ and $\xi \mathrm{i}$ are 0 , and there is no contribution to the linear combination $\mathrm{W}$; if the point falls just on the margin, then the result of equation $|\gamma-S|-\epsilon$ is 0 ; if outside, the value of $\alpha$ is not 0 .

\subsection{Analysis of the Model}

According to the introductions about herring and mackerel from the official website of Scottish Sea Fisheries Statistics, we learn that both they are cold-water fish and live in areas with seawater temperatures of $0-20^{\circ} \mathrm{C}$. Their migratory routes are similar, so they can be regarded as the same category of fish when they start to migrate. Besides, fishing companies in Scotland normally go fishing for herring and mackerel from June to November, so of course they will go fishing at July.

Then we divide the Scottish area (located in the geographic range of $50^{\prime} 00^{\prime \prime} \mathrm{N}$ to $70^{\prime} 00^{\prime \prime} \mathrm{N}$, and $16^{\prime} 00^{\prime \prime} \mathrm{W}$ to $2^{\prime} 00^{\prime} \mathrm{E}$ ) into a $17^{*} 21$ matrix , and each single point is trained by support vector regression we mentioned before(the specific algorithm is shown in the appendix). Taking one point $(50 \circ \mathrm{N}, 0 \circ \mathrm{W})$ as an example, during our training of data, $1 \circ$ longitude and $1 \circ$ latitude area near it is one unit and also one rectangular grid in our figures, namely,the range from $\left(50^{\circ} \mathrm{N}, 0^{\circ}\right)$ to $(51 \circ \mathrm{N}, 1 \circ \mathrm{W})$. In this $17^{*} 21$ matrix, each point contains its geographical location, and seawater temperature information with the depth as 5 meters. Also taking the point $(50 \circ \mathrm{N}, 0 \circ \mathrm{W})$ as an example, we train its previous data of seawater temperatures at the depth of 5 meters with each month in the past 20 years, and get its future seawater temperatures in nest 50 years with a depth of $5 \mathrm{~m}$. The figure is shown below: 

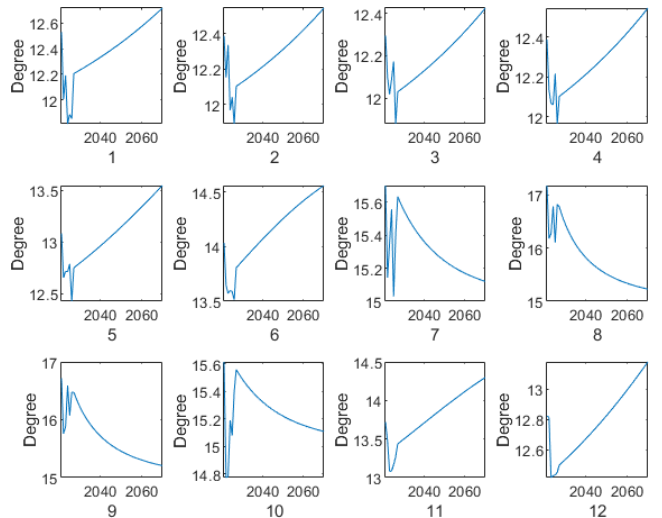

Figure2 $(50 \circ \mathrm{N}, 0 \circ \mathrm{W})$ Seawater temperatures from January to December after 50 years

After obtaining the future temperatures of each point in the matrix, we want to get the future temperatures of the whole area. In order to make the temperature prediction between points to points more accurate and smooth, we use the method of uniform interpolation between points, and give the best linear unbiased Prediction(BLUP) of future temperatures. We taking July in 2020 as an example, and show the results of it below:

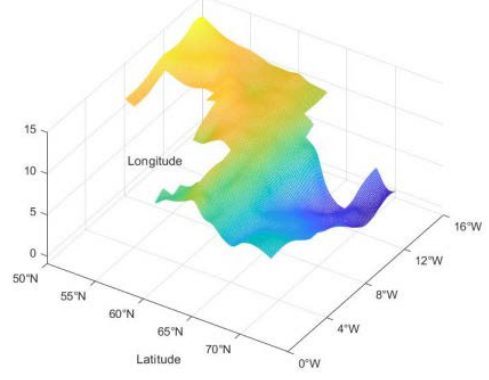

Figure3 3D temperature chart of July, 2020

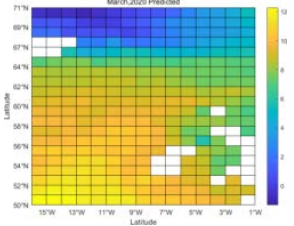

(a) March

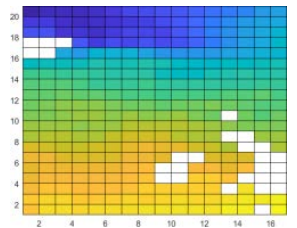

(b) June

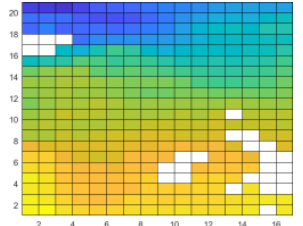

(c) September

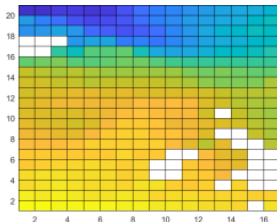

(d) December

Figure4 Temperature distribution map for four months

Then we look for areas with a target temperature $\left(4 \circ \mathrm{C}-15^{\circ} \mathrm{C}\right)$ on the monthly temperature distribution maps, which are the areas fishes most likely pass through, and also the main activity areas for the fishing companies. We also take 2020 as an example. During this year, the locations of the target temperature area are shown in the following figure.
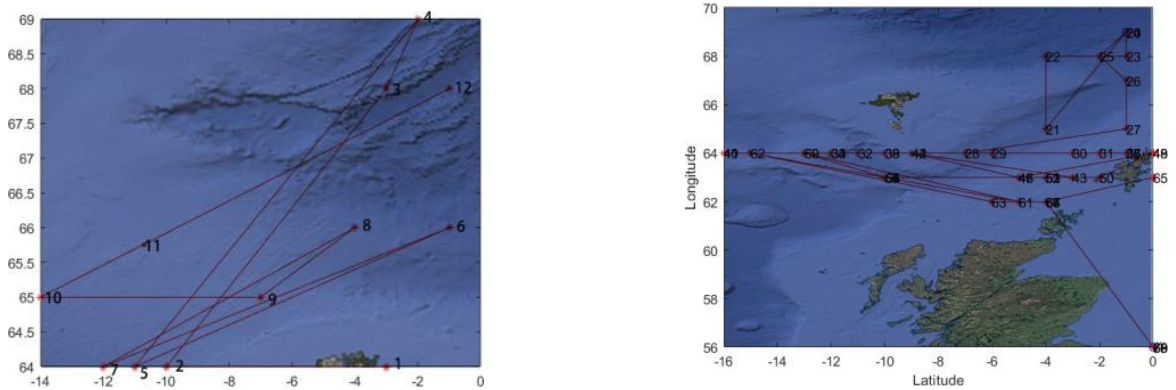

Figure5 Locations of target temperatures in 2020 Figure6 The migration locations of fish in next half century 
(*annotation: The dot represents the location of the target temperature, and the number next to it represents the month (1: January)). Finally, we take the target temperature region in July to represent the target temperature region of that year, and combine the temperature changes in the next 50 years predicted before to obtain the most likely positions for fish population migration in the next half century:

\subsection{Results of the Model}

The previous forecast of the target area in the next 50 years shows that two fishes are slowly moving towards the Arctic, which is caused by the global warming and continuous rising temperature of the ocean. But in picture 1.6, we are not easy to see this trend, so we are not sure about our results. Therefore, based on our previous forecast of the temperature distribution of the North Atlantic Ocean in the next 50 years, we use the geographic information system analysis tool ARCGIS to more accurately predict the change of the target temperature, which is also the change of the migration positions of two populations of fish.

Firstly, we find the geographical coordinates of the locations of the target temperature, and output the raster data directly from MATLAB, imported it into ARCGIS, and then find out the isotherms of the target temperature for five years (2020, 2025,2030,2040,2050 and 2060) by Kriging interpolation. Finally, we superimpose our results upon the map of the Scottish waters. The figure is shown below.

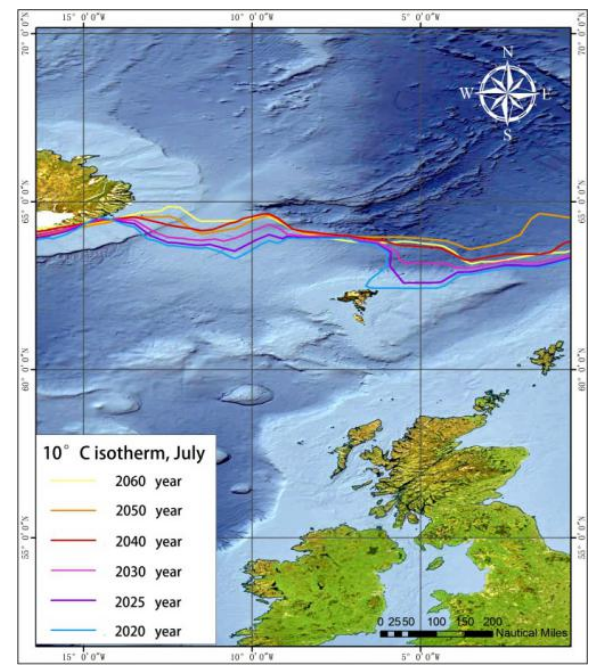

Figure $710 \circ \mathrm{C}$ Isotherm moving trend chart

From this figure, we can more intuitively see that as time goes by, the $10 \circ \mathrm{C}$ isotherm will gradually move northwards in the next 50 years. This is consistent with the results obtained by our SVM model, and to some extent, this figure embodies the effects of global warming on ocean temperatures.

\section{Companies Operation Evaluation Model}

\subsection{Construction Of The Model}

We find the data from Scottish sea fisheries statistics report in 2016 (Marine Scotland Directorate 2017) , and get that the fishing vessels generally owned by small fisheries companies are 500 tons, and the sailing speed of a ship that can carry about 20 crew members is about $0.864 \mathrm{~km} / \mathrm{min}$. The short-to-medium-distance voyage normally takes 2 days, with a voyage of 5 hours one day, and the rest of time is used for rest and processing the salvaged fish. So we can calculated the maximum fishing range for this type of fishing vessels is:

$$
R=V \times T=259.2 \mathrm{~km}
$$

Then on the map of Scotland we find out a famous port city named Kirkwall (62॰00 '35.03 "N, $2 \circ 46^{\prime} 17.90^{\prime \prime} \mathrm{W}$ ) in the north of Scotland, where there are fishing companies of all sizes. If there is no effect of extreme weather such as typhoons, fishing companies will go out to sea for fishing in the 
fishing season. We combine the prediction of changes in the target area in each year in Model 1, and take the location of the port as the center of the circle, the maximum sailing distance of small-sized fishing companies as the radius, and draw the sailing range of the fishing boat. We can regards the areas of the points inside the circle as where the small fishing companies can harvest Herring and mackerel:

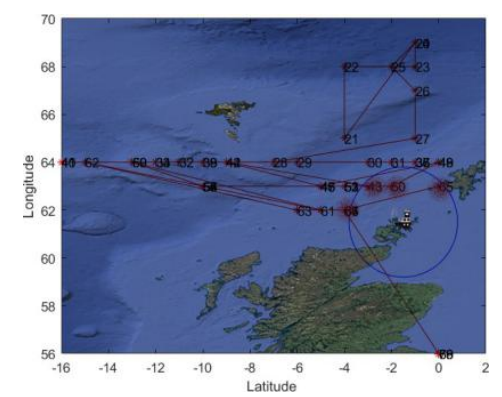

Figure8 The Fishing area for small fishery companies

\subsection{Analysis of the Model}

\subsubsection{Best Case}

As global warming becomes more severe, glaciers are melting, and sea levels are rising, we assume that the global warming trend will remain the same in the next 50 years for the best case. The chart in Figure 8 is the best fishing situation in the next 50 years. After 2065, the target area of two populations of fish will exceed the company's maximum fishing range. From then on, if the company continues to operate at the current location, it will not be able to make a profit on the fishing of these two types of fish.

\subsubsection{Worst Case}

If humans still pay little attention to environment protection, the carbon dioxide concern-tration in the atmosphere continue to rise, the speed of global warming will not slow, and the degree of water temperatures change will be more radical, which will lead to an expansion in the target area for catching fish. So we increase the speed of global warming by 1.5 times compared to the original speed predicted before, and re-simulate the changes in the target area of the fishes. We can witness the largest fishing area and the most likely locations of fish distribution in the next 50 years from the figure below:

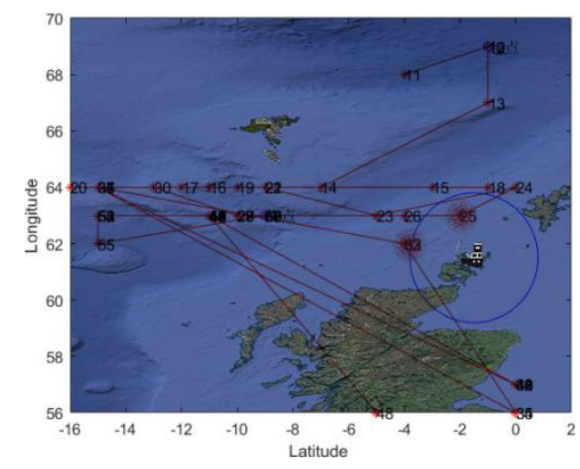

Figure9 The Fishing area for small fishery companies with 1.5 times global warming

As the figure above reveals, after 2032, it is highly possible that the target area of the fishes will exceed the company capability to voyage. After that, the small fishing companies will not be able to gain profits from harvesting herring and mackerel unless they adjust their operation strategies.

\subsection{Results of the Model}

According to the above analysis, small fishery companies can operate till 45 years later at best, and can only operate for 12 years at worst. After 12 years, the company can only fish by investing in new 
ships with longer sailing distances, but we know that this is very difficult for small fishing companies with limited financial resources.

\section{Transnational Fishing Activities}

Because fishery operations are irregular sea operations, people are likely to enter the high seas and other countries' territorial waters during fishing. So if it is necessary, they may even need to open branches in cities in other countries. However, they will be affected by local relevant policies and language differences. Both the analysis of the benefits of policy factors and language factors and the AHP method are used to determine the weight ratio of each influencing factor. According to maritime law and International Ocean law, outside the territorial sea of the country belongs to the high seas. Restricted by the innocent passage of foreign ships; the high seas is open to all countries, and the high seas are free. In terms of fisheries alone, coastal countries have exclusive fishing rights in their territorial waters, and other countries have no right to fish in the territorial waters of coastal countries. Some coastal countries prohibit foreign countries from engaging in fishing activities in their territorial waters, leaving all coastal fishing rights to their citizens. Most countries usually allow foreign vessels to fish in their territorial waters on the basis of fishing agreements or mutual benefits.

In order to meet the universality of the model, we have adopted policies that allow other country to fish but with certain limitation. However, since the quality of the fish itself is a benchmark for measuring the effectiveness of the company, a 10-point expert scoring method is used. The freshness factor is set to 8 points and the policy factor is set to 7 points. The language and culture of different countries also affect the company's revenue, but the influencing factors are relatively small, so it is rated as 5 points, and the comparison matrix is as follows:

$$
\left(\begin{array}{lll}
1 / 1 & 8 / 7 & 8 / 5 \\
7 / 8 & 1 / 1 & 7 / 5 \\
5 / 8 & 5 / 7 & 1 / 1
\end{array}\right)
$$

According to the data, option B in the proposal (to transfer assets to establish a branch company) is still the most effective choice, but the policy and language-related factors will lead to a reduce in the revenue to a certain extent. To describe the prediction error,we suppose $\mathrm{T}$ is the sample after training, $\mathrm{O}$ is the original data, and $\mathrm{N}$ is the month.

$$
\left[\begin{array}{cc}
\operatorname{plan} A & 8,006,500 \\
\operatorname{plan} B & 4,829,600 \\
\operatorname{plan} C & 71,499,672
\end{array}\right]
$$

New weight vector $\mathrm{K} 1=0.4 ; \mathrm{K} 2=0.35 ; \mathrm{K} 3=0.25$. The weight vector $[0.45,0.3,0.2]$ in model three is adopted in the target area of the domestic sea area indicates that according to the United Nations Convention on the Law of the Sea, we have marked the maritime boundary of the Scottish region. We choose Tórshavn62 ${ }^{\circ} 00^{\prime} 35.03$ " N6 ${ }^{\circ} 46^{\prime} 17.90^{\prime}$ " Wand the previously mentioned port city of Kirkwall in northern Scotland as the starting point for the fishing trip, as shown below:

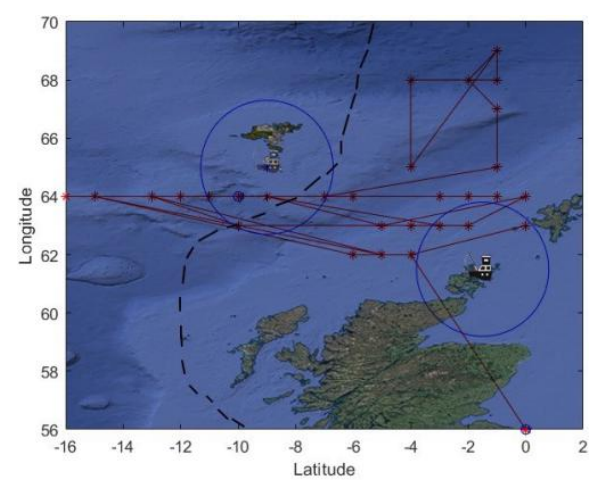

Figure10 Fishing ranges in different countries

Among them, the red dots are the most likely areas for fish and are the target areas for fishing companies; the blue circles represents the fishing capacity range of small and medium- sized fisheries 
companies. The company operating model established in Option 3 is used to calculate A and B The maximum returns of three cases, $\mathrm{C}$ are:

$$
\left[\begin{array}{cc}
\operatorname{plan} A & 8,006,500 \\
\operatorname{plan} B & 4,829,600 \\
\operatorname{plan} C & 71,499,672
\end{array}\right]
$$

According to the data, Option B in the proposal (to transfer assets to establish a branch company) is still the most effective choice, but the policy and language-related factors will lead to a reduce in the revenue to a certain extent.

\section{References}

[1] Tang Bo, Hou Yijun, Yin Yuqi, Hu Po. Joint inversion of three-dimensional temperature field in northern South China Sea using satellite remote sensing sea surface temperature and height [J]. Marine Science, 2019, 43(11): 1-10.

[2] Xu Lili, Tang Danling, Cai Lina. Study on the Influence of Sea Water Temperature and Sea Surface Height in Different Water Layers on Tuna Fishing Ground [J]. China Water Transport (Second Half Month), 2018, 18(12): 120-121+124.

[3] Li Ronghui. Study on Habits and Migration of Fish and Its Monitoring Techniques [D]. Guangxi University, 2013. 